\title{
Wie der Wahrheit ihre Bedeutung abgesprochen wird
}

\author{
Jean Martin
}

Dr. med., Mitglied der Redaktion

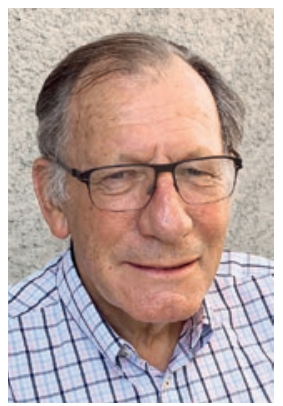

Literatur

1 Alain Campiotti, Le Temps, 7. Dezember 2020 , Seite 8 . Im Herbst 2020 veröffentlichte die Nachrichten-Website heidi.news einen aufschlussreichen Bericht über eine solche Gruppe in Genf.
Kürzlich unterhielt ich mich mit einem Kollegen vom CHUV über etwas, was man so nicht hätte erwarten können: wie sehr der Glauben an die - auf wissenschaftlichen Fakten basierenden - Konzepte von Wahrheit und Realität verloren gegangen ist.

Vor ein paar Jahren noch war mir nicht richtig bewusst, was da im Gange war - ich dachte, die Realität lässt sich doch belegen! Aber spätestens als Donald Trump, laut dem republikanischen Senator Mitt Romney ein Mensch, der es mit der Wahrheit nicht so genau nimmt ("who has a very loose relationship with the truth»), und laut einem anderen ein pathologischer Lügner («a pathological liar»), an die Macht gelangte, nahm dieses Phänomen weltweit so richtig an Fahrt auf. Trumps Umfeld hat dafür den doch sehr bedenklichen Ausdruck «alternative Fakten" (alternative facts) geprägt. Sorgen muss uns machen, dass fast die Hälfte der US-Bevölkerung sich an diese Lügen gewöhnt hat und, noch schlimmer, sie tatsächlich glaubt.

Im Namen der Freiheit (einfach alles sagen zu dürfen) wünschen sich manche, dass die Medien abweichenden Meinungen genauso viel Raum geben wie Fakten beziehungsweise den Einschätzungen anerkannt kompetenter Personen. Unter dem Titel «Les infortunes de la vérité» - Vom Problem der Wahrheit - erinnert uns ein altgedienter Journalist daran: «Nicht alle Meinungen sind gleichrangig. Es gilt, sich den Fakten zu beugen, die nun einmal so sind, wie sie sind» [1].

Aufgabe der Medien ist es, die gesellschaftlichen Verwerfungen objektiv zu beobachten, sie zu schildern und fundiert $\mathrm{zu}$ kommentieren. Aber genau das geschieht eben nicht in sozialen Netzwerken, wo den abstrusesten Behauptungen genauso viel Gewicht beigemessen zu werden scheint, wenn nicht sogar mehr, wie der Meinung einer Gruppe von Expertinnen und Experten (denken wir hier nur an das Intergovernmental Panel on Climate Change IPCC zum Thema Klima) oder den Einschätzungen von Amtsträgern und Führungskräften, die eine Situation im Hinblick auf deren Ursprünge und Konsequenzen beurteilen und handhaben müssen.

Natürlich sind auch die Meinungen von Expertinnen oder «Weisen» zu hinterfragen, aber von kritischen Stimmen sollte man doch ein Mindestmass an Objekti- vität und gesundem Menschenverstand erwarten können. Covid-19 hat zu einer Flut von Ideen und Behauptungen über verschiedene Aspekte der Pandemie geführt, in die oft Verschwörungsmythen einfliessen, bis hin zu Vorstellungen unterirdischer Machenschaften, die den Weltenlauf fernsteuern. Wir würden demnach also massiv manipuliert, und die meisten unter uns bekämen gar nichts davon mit. Insgesamt gesehen ist der Erfolg von Bewegungen wie QAnon sehr beunruhigend [2].

Das Problem: Selbst den wirrsten Behauptungen wird gerne Glauben geschenkt, wenn sie nur souverän genug und in klarem Gut-böse-Schema vorgebracht werden. So wurde das sich als Dokumentarfilm gerierende Video Hold up, eine Ansammlung unfundierter und vereinfachender Thesen, im Internet unzählige Male angesehen. Doch das Leben und unsere Welt sind komplex - was der französische Philosoph Edgar Morin seit Jahrzehnten $\mathrm{zu}$ verdeutlichen versucht. Und komplexe Situationen verwirren uns oftmals. Für Entscheidungsträger und andere Verantwortliche stellt dies immense kommunikative Herausforderungen dar. Diese Problematik hat dem Bundesrat und den Kantonsregierungen im Jahr 2020 wiederholt Kopfzerbrechen bereitet.

Ein aktuelles Beispiel ist die Impfung gegen Covid. Alle, die sich bisher öffentlich geäussert haben, sprechen sich gegen eine Impfpflicht aus. Nun aber wurde eine Petition gestartet, den Verzicht auf eine Impfpflicht in der Verfassung zu verankern. Als gelte es, einem Komplott entgegenzuwirken. Ein Sturm im Wasserglas à la suisse ... Die Unterzeichner verlangen insbesondere, eine unterschiedliche Behandlung von Impfverweigerern zu untersagen. Auf den ersten Blick eine vernünftige Forderung. Aber was wird die Eidgenossenschaft tun können, wenn eine Airline vor Flugantritt oder ein anderes Land bei der Einreise einen Impfnachweis verlangt?

Wenn - auf der Basis verlässlicher Daten - verhindert werden soll, dass nicht geimpfte Menschen andere ernsthaft gefährden, können auch solche als «diskriminierend» empfundene Massnahmen sinnvoll sein. Das Leben in der Gesellschaft erfordert es, ein vernünftiges Gleichgewicht zwischen den Rechten und Pflichten der Bürgerinnen und Bürger zu finden. Einmal mehr ist es wichtig, verständliche Informationen zur Hand zu haben und diese überzeugend zu vermitteln! 